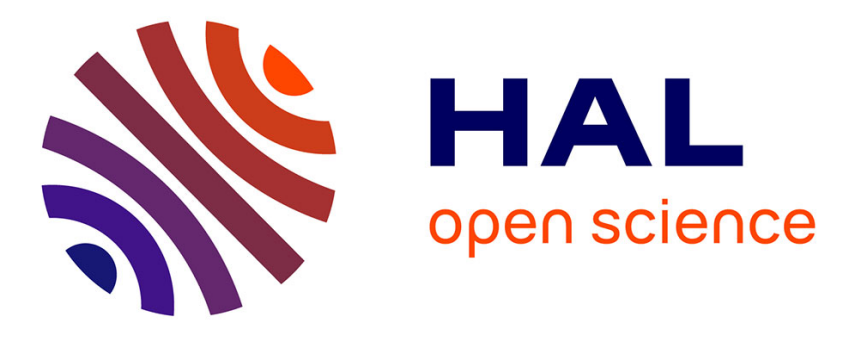

\title{
Les soins primaires : une définition du champ pour développer la recherche
}

\author{
Mélanie M. Afonso, Antoine Bénard, Anthony Chapron, Kenora Chau, Eric \\ Doussiet, Francis Guillemin, Sylvie Guillo, Damien Gonthier, Sophie Lafarge, \\ Mestre Lapeyre-Mestre, et al.
}

\section{To cite this version:}

Mélanie M. Afonso, Antoine Bénard, Anthony Chapron, Kenora Chau, Eric Doussiet, et al.. Les soins primaires : une définition du champ pour développer la recherche. Epidemiology and Public Health = Revue d'Epidémiologie et de Santé Publique, 2018, 66 (2), pp.157-162. 10.1016/j.respe.2017.09.004 . hal-01708871v2

\section{HAL Id: hal-01708871 \\ https://hal.science/hal-01708871v2}

Submitted on 27 Sep 2018

HAL is a multi-disciplinary open access archive for the deposit and dissemination of scientific research documents, whether they are published or not. The documents may come from teaching and research institutions in France or abroad, or from public or private research centers.
L'archive ouverte pluridisciplinaire HAL, est destinée au dépôt et à la diffusion de documents scientifiques de niveau recherche, publiés ou non, émanant des établissements d'enseignement et de recherche français ou étrangers, des laboratoires publics ou privés. 
Titre français : Les soins primaires : une définition du champ pour développer la recherche

Titre anglais: Primary care: a definition of the field to develop research

Titre courant : Définition du champ des soins primaires

Auteurs : Le groupe «Soins primaires » du réseau RECaP (Recherche en Epidémiologie

Clinique et en Santé Publique)

\section{Membres du groupe :}

M. Afonso, CIC-EC 1401, Bordeaux

A. Bénard, CIC-EC 1401, Bordeaux

A. Chapron, CIC-P 1414, Rennes

K. Chau, CIC-EC 1433, Nancy

E. Doussiet, CIC-EC 1410, Saint-Pierre de la Réunion

F. Guillemin, CIC-EC 1433, Nancy

S. Guillo, CIC-P 1421, Paris

D. Gonthier, CIC-EC 1433, Nancy

S. Lafarge, CIC-EC 1410, Saint-Pierre de la Réunion

M. Lapeyre-Mestre, CIC-P 1436, Toulouse

M. Oriol, CIC-EC 1408, Saint-Etienne

F. Tubach, CIC-P 1421, Paris

A. Verga-Gérard, CIC-EC 1433, Nancy

Affiliation : Réseau RECaP, Inserm

Coordonnés de l'auteur en charge de la correspondance :

Amandine Verga-Gérard, chef de projet du réseau RECaP,

Téléphone : 03.83.85.93.02,

Fax : 03.83.85.12.05

Adresse électronique : a.verga-gerard@chru-nancy.fr

https://dx.doi.org/10.1016/j.respe.2017.09.004 


\begin{abstract}
Research in the field of primary care has dramatically increased in France in the recent years, especially since 2013 with the introduction of primary care as a thematic priority within calls for proposal launched by the Ministry of Health (Direction Générale de l'Offre de Soins).

The RECaP (Research in Clinical Epidemiology and Public Health) network is a French research network supported by Inserm, which recently implemented a specific working group focusing on research in primary care, based on a multidisciplinary approach. Researchers from different specialties participate in this group. The first aim of the group was to reach a common definition of the perimeter and of the panel of health care professionals and structures potentially involved in the field of primary care. For this purpose, a selection of different data sets of sources defining primary care was analyzed by the group, each participant collecting a set of sources, from which a synthesis was made and discussed.

A definition of primary care at different levels (international, European and French) was summarized. A special attention was given to the French context in order to adapt the perimeter to the characteristics of the French health care system, notably by illustrating the different key elements of the definition with the inclusion of primary care actors and the type of practice premises.
\end{abstract}

In conclusion, this work illustrates the diversity of primary care in France and the potential offered for research purposes.

Key words: Primary care, General practice, Public Health 


\section{Résumé}

La recherche en soins primaires se développe depuis plusieurs années en France, particulièrement depuis 2013 avec la mention explicite des soins primaires comme priorité thématique des appels à projets de recherche sur les soins et l'offre de soins de la Direction Générale de l'Offre de Soins.

Le réseau RECaP (Recherche en Epidémiologie Clinique et en Santé Publique) est un réseau national de recherche, soutenu par l'Inserm. Dans le cadre de son fonctionnement, le réseau a mis en place des groupes de travail thématiques dont le groupe « Soins Primaires ». Des chercheurs issus de disciplines différentes participent à ce groupe. En conséquence, une des premières réflexions du groupe a été la nécessité d'utiliser des éléments de langage communs, basée sur une définition consensuelle des soins primaires. Pour cela, l'ensemble des participants au groupe a sélectionné différentes sources définissant les soins primaires à partir desquelles un état des lieux a été réalisé et discuté.

La définition des soins primaires à différentes échelles (internationale, européenne et française) a ainsi fait l'objet d'une synthèse. Une attention particulière a été apportée à la définition des soins primaires pour le contexte français afin de l'adapter aux particularités du système de soins français, notamment en illustrant les différents éléments clés de la définition des soins primaires par l'identification des acteurs et des lieux d'exercices associés.

En conclusion, ce travail illustre la variété et la richesse des soins primaires et permet ainsi de réfléchir au développement de la recherche dans ce domaine.

Mots clés : Soins primaires, Médecine générale, Santé Publique 
La recherche en soins primaires se développe depuis plusieurs années en France. Le comité d'interface Inserm (Institut National pour la Santé et la Recherche Médicale) Médecine générale qui existe depuis 2000 en est l'une des illustrations. Par ailleurs, les rapports à destination des ministres de la santé «Développer la recherche en médecine générale et en soins primaires en France : Propositions » de G. de Pouvourville en 2006 (1) et « La place et le rôle de la Médecine générale dans le système de santé » de P-L. Druais en 2015 (2) ont souligné qu'il est indispensable de développer une politique nationale de recherche en soins de santé primaires. Au niveau gouvernemental, les soins primaires sont une priorité depuis la mise en place de la stratégie nationale de santé en 2013 (3). Ainsi, depuis cette date, les soins primaires font partie des priorités thématiques des appels à projets de recherche sur les soins et l'offre de soins lancés par la Direction Générale de l'Offre de Soins (DGOS). Dans la circulaire $\mathrm{N}^{\circ}$ DGOS/PF4/2016/382 du 9 décembre 2016, les soins primaires sont définis ainsi : «Les soins primaires englobent les notions de premier recours, d'accessibilité, de coordination, de continuité et de permanence des soins. Les soins primaires constituent la porte d'entrée dans le système qui fournit des soins de proximité, intégrés, continus, accessibles à toute la population, et qui coordonne et intègre des services nécessaires à d'autres niveaux de soins. S'ils sont le premier contact des patients avec le système de soins, les soins primaires sont également structurant pour la suite du parcours du patient au sein du système de santé » (4).

Le réseau RECaP est le réseau national français de Recherche en Epidémiologie Clinique et en Santé Publique soutenu par l'Inserm (http://recap-inserm.fr) auquel participent notamment 13 Centres d'Investigation Clinique (CIC). Dans le cadre de son fonctionnement, ce réseau a mis en place des groupes thématiques de travail dont le groupe «Soins Primaires ». Ce groupe s'intéresse aux différentes problématiques, notamment méthodologiques, posées par la recherche en soins primaires et regroupe des chercheurs issus 
de différentes disciplines dans le champ des sciences de la santé. Du fait de ces cultures de recherche différentes, une des premières réflexions a été la nécessité d'avoir, pour le groupe, une définition consensuelle du champ des soins primaires, des éléments de langage communs. Cela est apparu comme une première étape avant la mise en place de projets de recherche collaboratifs répondant notamment à l'agenda de recherche en soins primaires proposés par le réseau européen de recherche en médecine générale (5). Pour cela, les membres du groupe ont identifié les différentes définitions des soins primaires par une revue narrative de la littérature (overview) au sens de Grant et al. (6). Les moteurs de recherche PubMed, et Google Scholar ont été utilisés. Les sites internet des sociétés savantes de médecine générale ont été consultés comme par exemple celui de la WONCA Europe (World Organization of National Colleges, Academies and Academic Associations of General Practitioners/Family Physicians). La recherche documentaire a été limitée aux sources anglophones et francophones, sans limite de dates de publications. Les auteurs ont ainsi sélectionné un ensemble de documents synthétisés par la chef de projet du réseau RECaP (auteur en charge de la correspondance). Cette synthèse a ensuite été discutée par l'ensemble du groupe lors de quatre réunions téléphoniques. La résolution des désaccords a été effectuée par reformulation puis consensus entre les coauteurs. Cet article est le résultat de ce travail.

\section{A l'échelle internationale}

Dans la déclaration d'Alma-Ata issue de la conférence du même nom organisée en septembre 1978, l'Organisation Mondiale de la Santé (OMS) indiquait que les soins de santé primaires sont «le premier niveau de contact des individus, de la famille et de la communauté avec le système national de santé, rapprochant le plus possible les soins de santé des lieux où les gens vivent et travaillent, et ils constituent le premier élément d'un processus ininterrompu de protection sanitaire. »(7). Dans cette déclaration, la vision des 
soins primaires présentée est une vision globale comprenant l'accès aux soins pour tous mais également l'amélioration des conditions de vie à un niveau satisfaisant (accès à l'eau potable, à l'alimentation, à l'éducation, ...). Le concept de soins primaires est porteur d'une ambition de justice sociale visant à garantir l'accès de tous à des soins de base. De cette définition initiale a découlé différentes interprétations et stratégies d'application selon les politiques locales. Cela est illustré par le rapport de l'Institut de Médecine américain «Primary Care: America's Health in a New Era », publié en 1996 où les soins primaires sont définis comme « des soins de santé intégrés, accessibles, réalisés par des médecins prenant en charge une grande majorité des besoins personnels de soins, développant une relation soutenue avec le patient et pratiquant dans le contexte de la famille et de la communauté » (8) ou encore par le rapport «Brazil's primary care strategy » de la Banque Mondiale qui présente un état des lieux des stratégies mises en place au Brésil afin de permettre à tous l'accès aux soins (9).

En 2007, l'agence régionale des Amériques de l'OMS (PAHO) publiait un article d'opinion, soulignant l'importance de renouveler la définition afin d'atteindre le but ultime des soins de santé primaire : des gains de santé durables pour tous (10). En 2008, l'OMS a publié un nouveau rapport sur la santé dans le monde consacré aux soins de santé primaires intitulé «Soins primaires : Maintenant plus que jamais » (11). Ce rapport propose un bilan de l'évolution des soins de santé dans le monde, pour réaffirmer les valeurs et objectifs prônés en 1978 et surtout pour rappeler que les besoins de la société évoluent selon des changements sociaux et environnementaux et qu'il est nécessaire de réorienter les systèmes de santé afin que l'offre de soins soit adaptée à la demande ainsi que cela était indiqué dans la déclaration d'Alma-Ata, «les soins primaires sont le moyen qui permettra d'atteindre l'objectif d'un niveau de santé qui permette aux peuples du monde de mener une vie socialement et économiquement productive »(7). 
En 2016, l'OMS indiquait sur son site internet que l'objectif ultime des soins de santé primaires est une meilleure santé pour tous (12). Cet objectif regroupe différents éléments clés : la réduction de l'exclusion et des disparités sociales dans le domaine de la santé, l'intégration de la santé dans tous les secteurs, l'organisation des services de santé autour des besoins et des attentes des populations et la participation des acteurs concernés dans des modèles de collaboration et de dialogue politique. Ces différents éléments ainsi que d'autres définitions telle que celle d'un système de soins basé sur les soins primaires sont consultables depuis le glossaire de l'OMS (13).

De cette définition, proposant une vision globale de la politique sanitaire mondiale, ont découlé plusieurs interprétations de la notion de soins primaires. En 2010, Friedberg et al proposaient trois définitions des soins primaires, fonction de leur modèle de mise en œuvre

- Les soins primaires comme spécialité : cette définition repose sur la « détermination des professions en charge des soins primaires, généralement les médecins généralistes, les médecins de famille, les pédiatres, les pharmaciens, les infirmières... »(15)

- Les soins primaires comme un ensemble de fonctions assurées par les offreurs habituels de soins : cette définition reprend celle de Barbara Starfield (16). Il s'agit des soins reçus par les patients lors de leur premier contact avec le système de santé, sur un territoire de proximité. Ces fonctions englobent le premier recours, l'approche globale du patient, la continuité des soins et la coordination avec les autres secteurs. Les fonctions sont alors indépendantes des formations et des qualifications professionnelles.

- Les soins primaires comme orientation des systèmes de santé : cette définition s'appuie sur l'organisation des parcours de soins et sur l'accessibilité des soins. 
Cartier et al ont proposé en 2012, un tableau (tableau 1) récapitulant les points communs des différentes définitions des soins primaires existantes (17).

- Accessibilité physique, géographique, temporelle et financière.

Premier contact - Aide au patient dans sa trajectoire de soins.

- Accueil sans aucune discrimination de tous les âges de la vie, de toutes les conditions de vie et de toutes les populations

- Garantie d'une absence d'interruption de la prise en charge des patients

Continuité des ou des communautés dans le temps. L'instauration d'une relation de soins confiance durable entre soignants et patients est un des critères majeurs de la qualité des soins.

- Situation au début de la chaîne médicale de prise en charge, coordination

Coordination des différents maillons, intersection de la prise en charge individuelle et des programmes collectifs de santé publique

- Prise en charge de l'individu dans sa globalité, sur le plan biologique, psychologique et social.

Globalité de la • Pas de focalisation sur un aspect du soin à l'inverse des soins secondaires prise soins en et tertiaires.

charge $\quad$ Prise en compte de la dimension communautaire. Proposition d'un large éventail de services de premier recours capables de répondre à la plupart des besoins sanitaires d'une population.

Tableau 1 : Socle commun de la définition des soins primaires (Traduction de Starfield par Cartier et al $(17,34))$ 


\section{A l'échelle européenne}

Plusieurs sources permettent d'avoir une vision de la définition des soins primaires à l'échelle européenne dont l'OMS Europe et la WONCA Europe. S'il existe des différences entre les pays européens, les notions clés de la définition des soins primaires sont communes. Les soins primaires sont définis comme le premier contact au sein du système de santé, accessible à tous. Ils prennent en compte le patient dans sa globalité (dimensions physique, psychologique, sociale, culturelle et existentielle) avec une prise en charge dans le temps et coordonnée avec l'ensemble du système de soins. Les définitions de l'OMS Europe et de la WONCA Europe précisent également que les soins primaires doivent promouvoir la santé et le bien-être par la prévention et l'éducation thérapeutique $(18,19)$. Dans son rapport publié en 2015, «Assurer les soins primaires dans une Europe en mutation », l'OMS Europe analyse le mode d'organisation et de prestation des soins primaires dans les pays européens et présente des résumés structurés de la situation des soins de santé primaires dans 31 pays européens (18,20). En 2014, la Commission Européenne publiait un avis du comité d'experts sur les moyens d'investir efficacement dans la santé dans lequel les soins primaires sont définis comme « un accès universel, intégré, personnalisé, complet aux soins et aux services de la communauté, prodigués par une équipe de professionnels qui répondent à la majorité des besoins de santé. Ces services ont lieu dans le cadre d'un partenariat soutenu entre le patient et le soignant, dans le contexte de la famille et de la communauté et jouent un rôle central dans la coordination et le suivi des soins $\gg(21)$.

\section{A l'échelle française}

En France, la notion de soins primaires est longtemps restée non définie. En effet, plusieurs éléments clés de la définition de l'OMS des soins primaires étaient déjà intégrés en 
France à travers la mise en place de l'Assurance maladie depuis 1928 et du système de protection sociale depuis 1945.

La définition des soins primaires est apparue dans la loi Hôpital Patient Santé Territoire (HPST) de 2009 sous le terme de « soins de premiers recours » proposés dans un périmètre géographique de proximité. (22). Organisés par les agences régionales de santé, les soins de premiers recours sont définis dans le code de la santé publique (article L1411-11 (23) en lien avec l'article 36 de la loi HPST,) comme comprenant :

- La prévention, le dépistage, le diagnostic, le traitement et le suivi des patients ;

- La dispensation et l'administration des médicaments, produits et dispositifs médicaux, ainsi que le conseil pharmaceutique ;

- $\quad$ L'orientation dans le système de soins et le secteur médico-social ;

- L'éducation pour la santé.

En 2016, l'article 64 de la loi de modernisation de notre système de santé (24) ajoute au code de la santé publique l'article L1411-11-1 relatif à la création des équipes de soins primaires définies ainsi : «Une équipe de soins primaires est un ensemble de professionnels de santé constitué autour de médecins généralistes de premier recours, choisissant d'assurer leurs activités de soins de premier recours définis à l'article L. 1411-11 (23) sur la base d'un projet de santé qu'ils élaborent. Elle peut prendre la forme d'un centre de santé ou d'une maison de santé.

L'équipe de soins primaires contribue à la structuration des parcours de santé. Son projet de santé a pour objet, par une meilleure coordination des acteurs, la prévention, l'amélioration et la protection de l'état de santé de la population, ainsi que la réduction des inégalités sociales et territoriales de santé. »(25). 
Des évolutions sémantiques se rencontrent également depuis 2016 dans certaines sociétés savantes, préférant parler de «soins premiers » plutôt que de soins primaires afin de traduire plus fidèlement le concept de « primary care » (26).

Associé à la création des équipes de soins primaires, se retrouve la mise en place des maisons de santé. Les maisons de santé sont des lieux qui regroupent des professionnels de santé, un exercice des soins de premier recours et un projet de santé (27). Il s'agit d'un mode de fonctionnement qui se développe en France mais qui est déjà établi dans d'autres pays comme la Belgique ou les Etats-Unis $(28,29)$.

Depuis la réforme de 2004, l'accès aux soins en France est centré autour du médecin traitant et du parcours de soins coordonnés (30). Le but du parcours de soins coordonnés est de permettre un suivi médical coordonné et une prévention personnalisée avec une prise en charge des patients sur la durée et centralisée autour du médecin traitant. Le choix du médecin traitant doit être fait par tous les patients à partir de 16 ans. En 2017, la nouvelle convention médicale étend le dispositif du médecin traitant aux enfants dès leur naissance (31). Dans la majorité des cas, le médecin traitant est un médecin généraliste mais ce n'est pas une obligation. C'est le médecin traitant qui oriente vers des soins spécialisés à l'exception des spécialités suivantes qui restent possible d'accès direct : la gynécologie, l'ophtalmologie, la stomatologie, la psychiatrie jusqu'à 25 ans et les soins effectués en urgence (32).

Cet état des lieux montre que si l'angle choisi pour définir les soins primaires peut différer, les différentes définitions se complètent et ne se contredisent pas. Ce travail nous a amené à proposer une illustration de la variété des acteurs et des lieux d'exercices des soins primaires en France. S'interroger sur les acteurs et les lieux permet d'ouvrir le champ de la recherche en soins primaires au-delà de la médecine générale, bien qu'elle en soit actuellement la discipline centrale (33). Nos échanges ont conduit à une adaptation du tableau 
de Cartier et al (17) (Tableau 2). Les professions (Qui ?) et les lieux (Où ?) associés aux différents éléments de la définition ont été discutés par le groupe et ajoutés en correspondance des colonnes «Caractéristique » et «Définitions » inchangées par rapport au tableau 1. Ces ajouts permettent de visualiser la richesse des possibles pour les soins primaires en France et de réfléchir ainsi au développement de la recherche associée à ce domaine en identifiant au mieux les acteurs et les lieux d'actions des soins primaires. 


\begin{tabular}{|c|c|c|c|}
\hline \multirow[t]{2}{*}{ Caractéristique } & Définitions & Profession associées (Qui ?) & \multirow{2}{*}{$\begin{array}{l}\text { Lieux associés (Où ?) } \\
\text { • Cabinet libéral }\end{array}$} \\
\hline & & & \\
\hline & & - Professionnels de santé prescripteurs : & - Maison de santé pluridisciplinaire \\
\hline & & - Médecin généraliste & - Pôle de santé \\
\hline & - Accessibilité physique, & - Dentiste & - Officines \\
\hline & géographique, temporelle et & - Sage-femme & - Services d'urgences \\
\hline & financière. & - Pharmacien & - Permanence de garde \\
\hline & - Aide au patient dans sa & - Médecin spécialiste accès direct & - Service de santé universitaire \\
\hline \multirow{9}{*}{ Premier contact } & trajectoire de soins. & (psychiatre, pédiatre, gynécologue, & - Service de santé pénitentiaire \\
\hline & - Accueil sans aucune & ophtalmologiste) et hors parcours de & - SOS Médecins \\
\hline & discrimination de tous les âges & soins & - Structures associatives et humanitaires \\
\hline & de la vie, de toutes les & - Sage-femme & (Médecins du monde, ,...) \\
\hline & conditions de vie et de toutes & - Professionnels paramédicaux dont & - Centre de dépistage anonyme et gratuit \\
\hline & les populations & - Infirmière diplômée d'état & - Centre de Planification et \\
\hline & & - Masseur-Kinésithérapeute & d'Education Familiale \\
\hline & & - Puéricultrices & - Laboratoire d'analyses \\
\hline & & & - Protection maternelle et infantile (PMI) \\
\hline
\end{tabular}




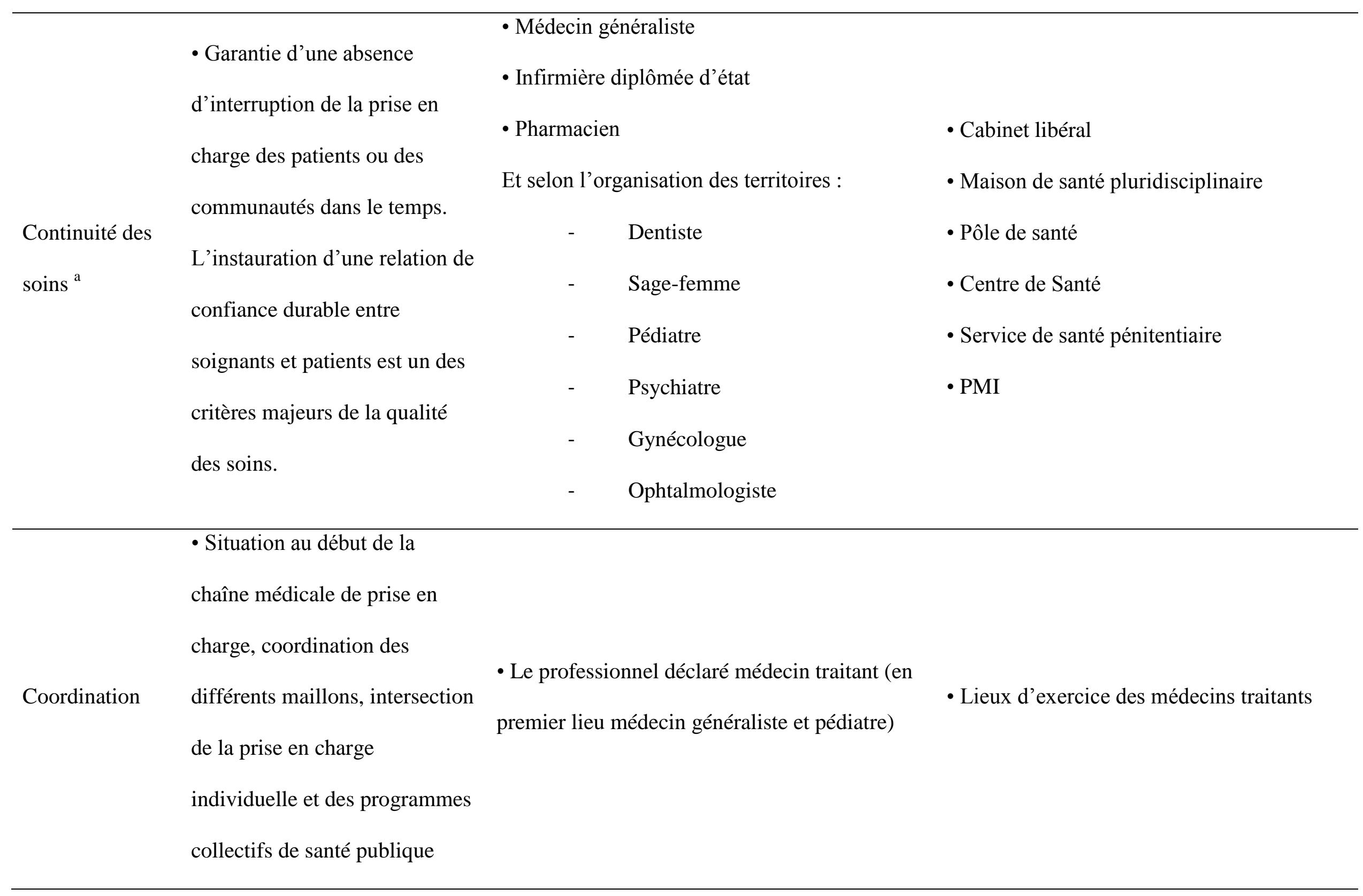


- Prise en charge de l'individu

dans sa globalité, sur le plan

biologique, psychologique et

social.

- Pas de focalisation sur un

aspect du soin à l'inverse des

soins secondaires et tertiaires.

prise soins en

- Prise en compte de la

charge
- Médecin généraliste

- Infirmière diplômée d'état

- Pédiatre

dimension communautaire.

- Cabinet libéral

- Maison de santé pluridisciplinaire

- Pôle de santé

- Centre de Santé

Proposition d'un large éventail

de services de premier recours

capables de répondre à la

plupart des besoins sanitaires

d'une population.

Tableau 2 : Définition des soins primaires prenant en compte les professions et les lieux d'exercices

a Les choix faits ici sont fonction des lieux d'exercices 
Remerciements : les auteurs remercient le docteur Thomas Cartier de les avoir autorisé à réutiliser son tableau «Socle commun de la définition des soins primaires ».

Conflits d'intérêts : Les auteurs déclarent ne pas avoir de conflits d'intérêts en relation avec cette communication.

\section{Bibliographie}

1. de Pouvourville G. Développer la recherche en médecine générale et en soins primaires en France : Propositions. 2006.

2. Druais P-L. La place et le rôle de la Médecine générale dans le système de santé [Internet]. 2015 mars [cité 21 févr 2017]. Disponible sur: http://socialsante.gouv.fr/ministere/documentation-et-publications-

officielles/rapports/sante/article/la-place-et-le-role-de-la-medecine-generale-dans-lesysteme-de-sante

3. Ministère des Affaires Sociales et de la Santé. Feuille de route de la Stratégie nationale de Santé [Internet]. 2013 sept. Disponible sur: http://social-sante.gouv.fr/IMG/pdf/SNSversion-longue.pdf

4. Ministre des Affaires Sociales et de la Santé. INSTRUCTION N DGOS/PF4/2016/382 du 9 décembre 2016 relative aux programmes de recherche sur les soins et l'offre de soins pour l'année 2017. [Internet]. déc 9, 2016. Disponible sur: http://circulaires.legifrance.gouv.fr/index.php?action=afficherCirculaire \&hit=1\&retourA ccueil $=1 \& \mathrm{r}=41600$

5. Hummers-Pradier E, Beyer M, Chevallier P, Cos X, Eilat-Tsanani S, Fink W, et al. Programme de recherche pour la médecine générale/médecine de famille et les soins primaires en Europe. exercer, la revue francophone de Médecine Générale. 2011;96:369.

6. Grant MJ, Booth A. A typology of reviews: an analysis of 14 review types and associated methodologies. Health Info Libr J. juin 2009;26(2):91 - 108.

7. OMS | Déclaration d'Alma-Ata sur les soins de santé primaires [Internet]. WHO. [cité 20 avr 2017]. Disponible sur: http://www.who.int/topics/primary_health_care/alma_ata_declaration/fr/

8. Medicine I of. Primary Care: America's Health in a New Era [Internet]. Washington, DC: The National Academies Press.; 1996 [cité 12 sept 2017]. Disponible sur: https://www.nap.edu/catalog/5152/primary-care-americas-health-in-a-new-era

9. Dmytraczenko T, Couttolenc B. Brazil's primary care strategy [Internet]. The World Bank; 2013 janv [cité 13 sept 2017] p. 1-40. Report No.: 74957. Disponible sur: 
http://documents.worldbank.org/curated/en/881491468020373837/Brazils-primary-carestrategy

10. Macinko J, Montenegro H, Nebot. Renewing primary health care in the Americas: a position paper of the Pan American Health Organization/World Health Organization (PAHO/WHO). Pan American Health Organization, Washington, DC; 2007.

11. OMS | Rapport sur la santé dans le monde 2008 - Les soins de santé primaires: maintenant plus que jamais [Internet]. WHO. [cité 20 avr 2017]. Disponible sur: http://www.who.int/whr/2008/fr/

12. OMS | Soins de santé primaires [Internet]. WHO. Disponible sur: http://www.who.int/topics/primary_health_care/fr/

13. WHO | Health Systems Strengthening Glossary [Internet]. WHO. [cité 13 sept 2017]. Disponible sur: http://www.who.int/healthsystems/hss_glossary/en/index8.html\#8

14. Friedberg MW, Hussey PS, Schneider EC. Primary Care: A Critical Review Of The Evidence On Quality And Costs Of Health Care. Health Aff. 5 janv 2010;29(5):766-72.

15. Bourgueil Y. Systèmes de soins primaires: contenus et enjeux. Revue française des affaires sociales. 22 oct 2010;(3):11-20.

16. Starfield B, Shi L, Macinko J. Contribution of Primary Care to Health Systems and Health. Milbank Q. sept 2005;83(3):457-502.

17. Cartier T, Mercier A, De Pouvourville N, Huas C, Ruelle Y, Zerbib Y, et al. Constats sur l'organisation des soins primaires en France. Exercer. 2012;101(101):65-71.

18. Assurer les soins primaires dans une Europe en mutation [Internet]. OMS/Europe. 2015 [cité 20 avr 2017]. Disponible sur: http://www.euro.who.int/fr/aboutus/partners/observatory/publications/studies/building-primary-care-in-a-changing-europe

19. The European Definition of GP / FM [Internet]. Wonca Europe. [cité 20 avr 2017]. Disponible sur: http://www.woncaeurope.org/gp-definitions

20. Assurer les soins primaires dans une Europe en mutation - Etudes de cas [Internet]. OMS/Europe. 2015 [cité 20 avr 2017]. Disponible sur: http://www.euro.who.int/_data/assets/pdf_file/0011/277940/Building-primary-carechanging-Europe-case-studies.pdf?ua=1

21. Expert Panel on Effective Ways of Investing in Health (EXPH). Definition of a frame of reference in relation to primary care with a special emphasis on financing systems and referral systems. European Union, Brussels; 2014 juill.

22. Loi $\mathrm{n}^{\circ}$ 2009-879 du 21 juillet 2009 portant réforme de l'hôpital et relative aux patients, à la santé et aux territoires. 2009-879 juill 21, 2009.

23. Code de la santé publique - Article L1411-11 [Internet]. Code de la santé publique janv 26, 2016.2 Disponible sur: https://www.legifrance.gouv.fr/affichCodeArticle.do;jsessionid=7159F0A07DACFBD9 
78D6D77393EFD943.tpdila13v_2?idArticle=LEGIARTI000031930722\&cidTexte=LE GITEXT000006072665\&dateTexte $=20170123$

24. Loi n ${ }^{\circ}$ 2016-41 du 26 janvier 2016 de modernisation de notre système de santé. 2016-41 janv 26, 2016.

25. Code de la santé publique - Article L1411-11-1 [Internet]. Code de la santé publique janv 26, 2016. Disponible sur: https://www.legifrance.gouv.fr/affichCodeArticle.do;jsessionid=7159F0A07DACFBD9 78D6D77393EFD943.tpdila13v_2?idArticle=LEGIARTI000031917874\&cidTexte=LE GITEXT000006072665\&dateTexte $=20170123$

26. CNGE Communication [Internet]. 2016 [cité 22 févr 2017]. Disponible sur: https://www.cnge.fr/media/docs/cnge_site/cnge/CNGE_com_mai_16.pdf

27. Qu'est ce qu'une Maison et un Pôle de Santé? [Internet]. Fédération Française des Maisons et Pôles de Santé. [cité 13 sept 2017]. Disponible sur: http://www.ffmps.fr/index.php/definitions/quest-ce-quune-maison-de-sante-et-un-polede-sante

28. Fédération des Maisons Médicales et des Collectifs de Santé Francophone. Maison médicale, le centre de santé intégrée [Internet]. 2016. Disponible sur: http://www.maisonmedicale.org/Maison-medicale-le-centre-de-sante.html

29. Agency for Healthcare Research and Quality (US Dept of Health and Human Services). Defining the primary care medical home (PCMH) | PCMH Resource Center [Internet]. Agency for Healthcare Research and Quality (US Dept of Health and Human Services). [cité 13 sept 2017]. Disponible sur: https://pcmh.ahrq.gov/page/defining-pcmh

30. Loi n ${ }^{\circ}$ 2004-810 du 13 août 2004 relative à l'assurance maladie [Internet]. aout, 2004. Disponible sur: https://www.legifrance.gouv.fr/affichTexte.do?cidTexte=JORFTEXT000000625158\&da teTexte $=\&$ categorieLien $=$ id

31. Arrêté du 20 octobre 2016 portant approbation de la convention nationale organisant les rapports entre les médecins libéraux et l'assurance maladie signée le 25 août 2016 [Internet]. oct 20, 2016. Disponible sur: https://www.legifrance.gouv.fr/eli/arrete/2016/10/20/AFSS1629881A/jo/texte

32. Arrêté du 22 septembre 2011 portant approbation de la convention nationale des médecins généralistes et spécialistes. sept 22, 2011.

33. Gay B. Repenser la place des soins de santé primaires en France-Le rôle de la médecine générale. Revue d’Épidémiologie et de Santé Publique. juin 2013;61(3):1938 .

34. Starfield B. Is primary care essential? The Lancet. 22 oct 1994;344(8930):1129-33. 